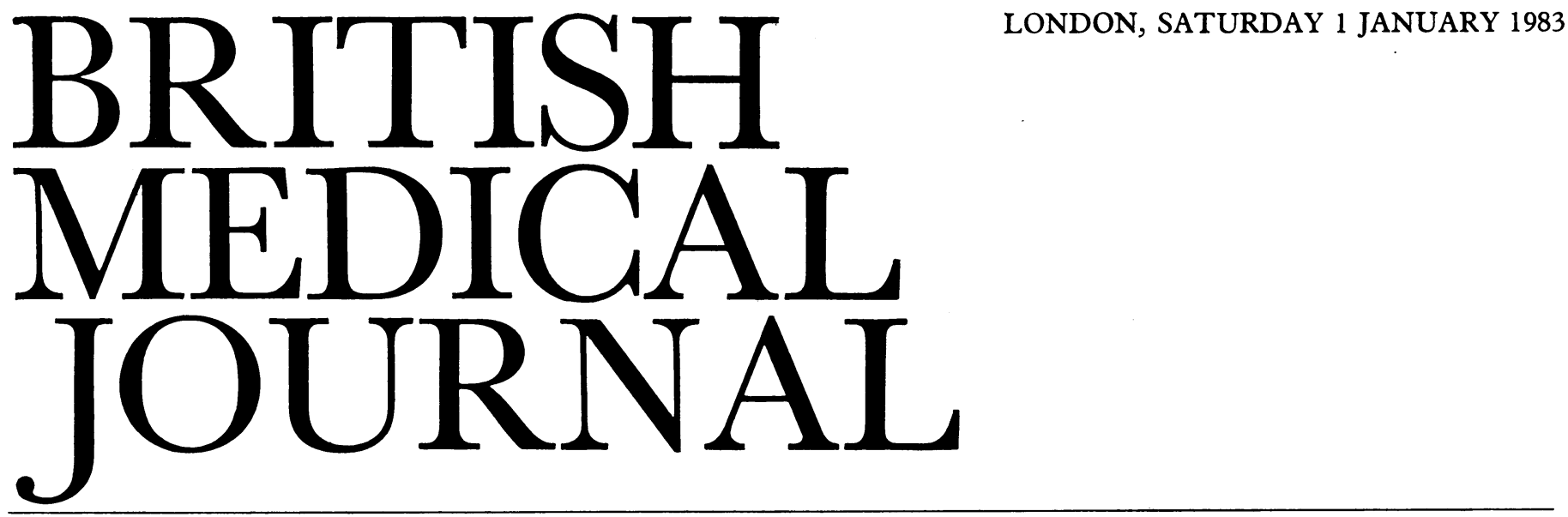

\title{
How will the new genetics work?
}

When $\mathrm{H} \mathrm{G}$ Wells returned from his first visit to the USSR he is said to have exclaimed "I have seen the future and it works." No one now claims to see the future so plainly, but Professor David Weatherall has provided a wide perspective of what may be coming in genetics. The facts are accessible to any reader willing to give time to his splendid monograph published in mid-December. ${ }^{1}$

The centrepiece of the recent explosion in knowledge of genetics is our new found ability to map and determine the fine structure of human genes and to define in molecular terms how each gene controls the structure of a single protein and so determines an essential characteristic of one or more tissues. What is the potential of the newer medical genetics for the wider control of human disease-and what problems may it bring in its wake?

One of the few certainties is that medical genetics is going to require ever more individuals to make difficult, sometimes draconian decisions about their children (either in being or planned). Two years ago lawyer Ian Kennedy used the Reith lectures to attack the caricature concept of the Olympian doctor giving authoritative advice to the acquiescent patient. ${ }^{2}$ Decision making in medicine should, he argued, be reformed to give the major role to the patient or consumer. Such a belief assumes that the facts underlying medical decisions can be presented to patients objectively and comprehensively. In reality, the doctor or counsellor who presents the facts rarely does so with total, dispassionate objectivity; and increasingly often the range and detail of the basic information is far wider than can easily be comprehended by the counsellor, let alone the patient.

Genetic disorders account for a substantial fraction of all human disease, but specific diseases due to single gene defects are mostly rare in Western societies. Ordinary clinicians see few patients with Huntington's chorea, polyposis coli, or the Marfan syndrome (inherited as Mendelian dominant disorders); with cystic fibrosis, phenylketonuria, or thalassaemia (inherited as recessive disorders); or with Duchenne muscular dystrophy or haemophilia (inherited as X-linked disorders). In some societies, however, these single gene defects are much more frequent; blindness and early death due to Tay Sachs disease may be as frequent as 40 in every 100000 births in Ashkenazic Jews and thalassaemia affects as many as one in 50 children in some Mediterranean countries.

Chromosomal abnormalities such as Down's syndrome and the sex chromosome disorders (Turner's syndrome, Klinefelter's syndrome) are more common in Western society than single gene defects; altogether chromosomal abnormali- ties are found in about 5.6 births per 1000. Congenital malformations such as spina bifida (two to eight per 1000 births) and congenital heart disease (six to eight per 1000 births) are more common still, but in these cases the genetic component is only partial, with the balance coming from environmental influences.

Finally, four common disorders-schizophrenia, manicdepressive psychosis, epilepsy, and diabetes mellitus-have a strong genetic component and many others have a less substantial genetic contribution. Overall, Professor Weatherall estimates that genetic disease and congenital malformations occur in approximately $2-5 \%$ of all live births, account for one third of paediatric admissions to hospital, and are the cause of $40-50 \%$ of deaths in childhood. Chronic diseases with an appreciable genetic component occur in about $10 \%$ of the adult population.

So much has been known for many years. The "new genetics" began with the precise mapping of human genes. Firstly, characteristics such as inherited disease or an enzyme activity may be mapped on to a single stained human chromosome; secondly, the mapping may move down to the molecular level, determining the sequence of the DNA composing the gene responsible for the disease or enzyme activity.

The two sorts of genetic maps are on very different scales. When a single chromosome is stained some 20 bands are shown up by the dye. A single chromosome contains 100 million base pairs of DNA, so that with the use of chromosome banding the spots identified as the site of a gene average five million base pairs apart. By contrast, the molecular biologist can study fragments of DNA only several hundred base pairs long. The section is done by "restriction" enzymes, which act only on certain sites in the base sequences of DNA. The fragments produced can then be inserted into the DNA of bacteria which can be cultured to produce large quantities of the fragment and its molecular structure can then be determined. Libraries are now being built up containing hundreds of thousands of different bacteria containing lengths of DNA each representing roughly one gene.

When the search moves on to the identification of genetic defects, even the mapping achieved by restriction enzymes leaves many questions unanswered. Mutations which lead to a single change in one of the nucleotides in a section of DNA may readily be detected by restriction enzymes if the change affects one of the sites at which these enzymes act. Direct recognition of an abnormal gene will be possible when the mutation provides a new site or eliminates an old one at which the enzyme splits the DNA or when the mutation causes a rearrangement 
of substantial size; in either case the effect is enough to change the gene map sufficiently to make the diagnosis plain. In most cases, however, those approaches do not lead to recognition of the abnormality-only a handful of the 300 abnormal haemoglobin genes can be pinpointed directly by restriction enzymes.

For the remainder, the answer seems likely to come from two difficult concepts-linkage and polymorphism. These are defined as follows. Linkage is the term used when the gene under study is found to be positioned very close to another gene which can be easily identified-for example, the associations between HLA antigens and genetic susceptibility to disease. Polymorphisms depend on the fact that many mutations alter the length of the fragments produced by restriction enzymes but have no other apparent effect. These neutral mutations produce variations - polymorphisms - in the fragment length produced by restriction enzyme analysis of the DNA in the normal population. If an identifiable change in the fragment lengths can be shown to be linked with an abnormal gene, then the two should be transmitted together genetically. Analysis of families with some members affected by the illness caused by the gene should then show whether the presence of the gene can be diagnosed by use of variation in the restriction enzyme fragment lengths.

Professor Weatherall warns that the establishment of linkages between fragment length polymorphisms and important medical genes is not going to be easy and that this diagnostic method seems unlikely to be very helpful in screening for carriers. Nevertheless he sees grounds for cautious optimism. $\mathrm{He}$ estimates that there may be around 10 million single-site restriction fragment length polymorphisms in the whole human genome, and there may be many highly variable regions which would allow linkage analysis in individual families.

Where is the new genetics leading us ? For the time being the main practical application is in prenatal diagnosis. Already the diagnosis of Down's syndrome, thalassaemia, and several other blood disorders has become an established part of clinical obstetrics-but obstetricians have to wait until well into the midtrimester before they can carry out procedures such as amniocentesis and fetoscopy and so obtain the cells or blood required. Recently, however, techniques developed in the USSR have been used to sample the trophoblast layer of the embryo at eight to 10 weeks' gestation. Professor Robert Williamson at St Mary's Hospital, London, has shown that enough DNA may be obtained in this way for direct analysis of the genes, so making possible the diagnosis of some haemoglobinopathies. Since, however, only a few haemoglobinopathies cause mutations directly identifiable by restriction enzymes this approach will be of limited value until more use can be made of restriction enzyme fragment length polymorphism. In most cases establishment of linkage between the abnormal gene and a specific polymorphism will depend on analysis of individual members of the family concerned. Ideally families should be examined at leisure-a time consuming and expensive undertaking.

Prenatal diagnosis and selective abortion may reduce the load of genetic disorders but they are surely, says Professor Weatherall, not the ultimate answers to genetic disease. But attempts to replace the missing enzyme-or even more ambitiously, the defective gene-have so far proved disappointing. The sequences of DNA that represent the healthy gene may be synthesised in bacterial culture and inserted into cells in cell culture-but beyond that lie vast problems. Professor Weatherall has again taken haemoglobinopathies as his example. Clearly the normal gene would have to be inserted into a haemopoietic stem cell; and stem cells are relatively rare in the bone marrow. Calculations suggest that 1000 million marrow cells (found in $25 \mathrm{ml}$ of bone marrow) would need to be treated to be certain of transferring a new gene into one stem cell using current techniques. But transfer of the new gene into a handful of stem cells would be unlikely to affect the disease, since the new population would not necessarily flourish at the expense of the old. Nor would the new genes necessarily function properly; the foreign gene might produce globin in white cells or platelets rather than red cells. Attempts made in mice to insert human globin genes into stem cells have not led to human beta globin chains being synthesised in mouse red cell precursors.

So far the one method proved to be effective in haemoglobinopathies is destruction of the abnormal marrow by drugs and irradiation and replacement with healthy bone marrow from a histocompatible sibling. Such treatment poses substantial ethical problems. How severe must be the threat to life before such a hazardous treatment is undertaken? An infant with thalassaemia may live 25 years with repeated transfusions. Is the shortened life span justification for a treatment with a real mortality?

Indeed, many of the ethical problems likely to be raised by medical genetics are both daunting and complex. Prenatal diagnosis and selective abortion have been accepted in the West even in Catholic countries such as Italy, but the response of Islamic communities is less certain. Experience with prenatal diagnosis of thalassaemia in London, Sardinia, and Cyprus, of Tay Sachs disease in the United States and Israel, ${ }^{3}$ and of sickle cell disease in the United States has shown that counselling and sympathetic public relations campaigning are vital. Identification of carriers of a disease may not necessarily be seen as beneficial by the community concerned, especially if the result is widespread "genetic anxiety." Indeed, the sickle cell disease programme in the United States foundered on accusations of racialism, and immigrant populations are likely to be especially sensitive on this issue.

So how far will patients, "consumers," and their families be able to take part in the decisions that will flow from the advances being made so rapidly in the new genetics? Firstly, says Professor Weatherall, clinical geneticists will have to be completely informed about the natural history of genetic disease, treated and untreated. Secondly, counsellors will have to be trained to present increasingly complex concepts in simple language. Finally, all concerned will have constantly to reevaluate what is meant by "quality of life" for handicapped children.

Ideally children should be taught about the underlying concepts so that they have some background knowledge on which to build as they approach parenthood. Above all, journalists should surely recognise the need to avoid sensationalism and racialist confrontation when discussing these issues; the dramatic reduction in the numbers of children being born with thalassaemia among Cypriots living in London shows what can be achieved by a programme combining good science with good information services for the community. ${ }^{4}$

TONY SMITH

Deputy Editor, $B M \mathcal{F}$

1 Weatherall DJ. The new genetics and clinical practice. London: Nuffield Provincial Hospitals Trust, 1982.

2 Anonymous. The Reith lectures: style, responsibility, and accountability. Br Med f 1980;281:1659-60.

3 Goodman MJ, Goodman LE. The overselling of genetic anxiety. Hastings Cent Rep 1982;12:20-7.

4 Modell B, Ward RHT, Fairweather DVI. Effect of introducing antenatal diagnosis on reproductive behaviour of families at risk for thalassaemia major. Br Med f 1980;280:1347-50. . . , (1)}

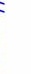

